\title{
Noise Radiation from a Continuous Mold-Line Link Flap Configuration
}

\author{
Florence V. Hutcheson*, Thomas F. Brooks, William M. Humphreys, Jr.** \\ Aeroacoustics Branch \\ NASA Langley Research Center \\ Hampton, Virginia 23681-2199
}

The results of an experimental study of the noise from a Continuous Mold-Line Link (CML) flap are presented. Acoustic and unsteady surface pressure measurements were performed on a main element wing section with a half-span CML flap in NASA Langley's Quiet Flow Facility. The acoustic data were acquired with a medium aperture directional array (MADA) of microphones. The Deconvolution Approach for the Mapping of Acoustic Sources (DAMAS) method is applied to determine the spatial distribution and strength of the noise sources over the surface of the test model. A Coherent Output Power (COP) method which relates the output from unsteady surface pressure sensors to the output of the MADA is also used to obtain more detailed characteristics of the noise source distribution in the trailing edge region of the CML. These results are compared to those obtained for a blunt flap to quantify the level of noise benefit that is achieved with the CML flap. The results indicate that the noise from the CML region of the flap is 5 to $17 \mathrm{~dB}$ lower (depending on flap deflection and Mach number) than the noise from the side edge region of the blunt flap. Lower noise levels are obtained for all frequencies. Spectral analysis of the noise from the cove region of the CML and blunt flap models also reveal a spectral peak in the high frequency range that is related to noise scattering at the trailing edge of the main element. The peaks in the CML and blunt flap cove noise spectra are close in level and often exceed blunt side edge noise. Applying a strip of serrated tape to the trailing edge of the CML flap model main airfoil, reduced the peak but increased other noise somewhat. Directivity measurements show that the CML flap can be more directional than the blunt flap.

\section{Introduction}

During airport approach when the engines of an aircraft are near idle and the high-lift systems and landing gear are deployed, airframe noise is a dominant noise source. The noise that is generated at the side edge of the flaps has been identified as an important component of airframe noise ${ }^{1-5}$.

A number of numerical and experimental studies ${ }^{6-13}$ have been conducted in order to identify and model the noise generation mechanisms at the flap side edge. Flow field measurements ${ }^{8}$ in the flap side edge region of a wing with a half-span flap have revealed the presence of a 2-vortex system (see Figure 1): a small vortex near the flap side edge on the top surface and a stronger side vortex along the lower portion of the flap side edge. As the side vortex travels downstream along the flap side edge, it strengthens and expands. At about mid-chord it begins to spill over to the flap top surface and merges with the small top vortex. The instabilities in this vortex system and in the strong shear layer that originates on the bottom edge of the flap create an unsteady pressure field at the flap side edge causing sound to radiate. Brooks et al. ${ }^{13}$ have determined that the dominant flap side edge noise regions are located around mid-chord on the pressure side of the flap edge and around $60-65 \%$ chord on the suction side.

Various flap side-edge noise reduction concepts have been evaluated. Experiments ${ }^{14,15}$ and CFD calculations ${ }^{16}$ showed that the use of a porous side-edge treatment led to the formation of a significantly weaker side-edge vortex system and the elimination of the bursting of the side edge vortex at large flap deflection angles. Both effects are expected to result in a decrease in the radiated noise. Experimentally, it has been shown that substantial noise reduction could be achieved by applying fence type devices ${ }^{17-18}$ to the flap tip. Koop et al. ${ }^{19}$ showed that flap tip winglets, and suction side fences were most effective in reducing flap side-edge noise and that pressure side fences and microtabs (vortex generators) were less effective.

\footnotetext{
* Research Engineer, AIAA Member

† Senior Research Scientist, AIAA Fellow

** Senior Research Scientist, AIAA Senior Member
} 


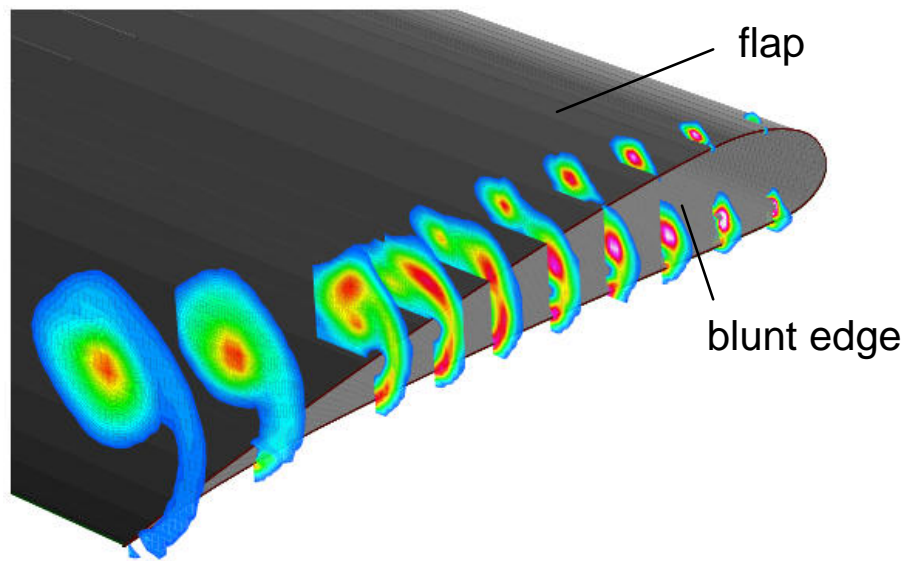

Figure 1. (from ref. 8) Axial vorticity contours shown at the blunt side edge of a half-span flap deployed at a $29^{\circ}$ angle with respect to the main element. Data are from 5 -hole probe measurements.

In the same study, Koop et al. also demonstrated an active flow control method ${ }^{19-20}$ for noise reduction. Air was blown into the flap side-edge vortex system through a series of small round orifices located along the flap suction and pressure side edges between 13 and 35\% chord. The blowing caused the vorticity in the flap side-edge shear layer to be concentrated in small vortices (located further away from the flap surface with increasing blowing momentum). The vorticity field on the suction side of the flap appeared to be more homogeneous and the interaction between the curved shear layer and the suction side edge was reduced. As a result, very good noise reduction was achieved between 2 and $5 \mathrm{kHz}$. Hutcheson et al. ${ }^{21}$ also investigated the effect of flap tip jets on flap-side edge noise. Air was blown through thin rectangular slots located near or along the flap side edge. Based on the known structure of the flap side-edge flow and on the afore-mentioned findings by Brooks et al. ${ }^{13}$, several tip jet configurations were designed. PIV (Particle Image Velocimetry) measurements and CFD calculations were performed to evaluate the effect of the tip jets on the flap side-edge flow field. The tip jet configurations that showed the best noise reduction potential were identified.

Another, more radical, approach to controlling flap side edge noise is to "eliminate" the flap side edge by fairing it into the trailing edge of the stationary main wing ${ }^{22}$. The extended, faired in, portions of the flap are referred to as a "continuous mold-line link", or CML. An example of a CML flap configuration is shown in Figure 2. The role of the CML is to prevent the formation of the strong and concentrated shear-layer/vortex system that is present at the blunt flap side edge, and to replace it with a more "diffused" system by distributing the spanwise lift discontinuity across the CML region.

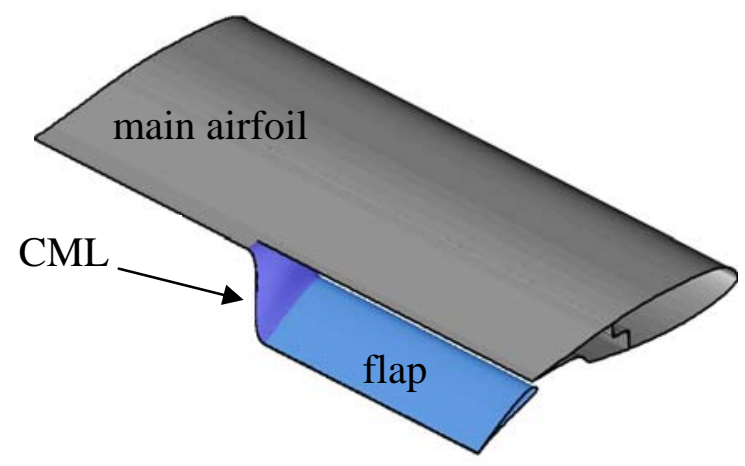

Figure 2. Sketch of a wing section with a Fowler flap and CML.

Storms et al. ${ }^{23}$ tested the CML concept on an hinged flap configuration, representative of proposed BlendedWing-Body (BWB) aircraft design ${ }^{24}$ for which the use of a more complex (slotted flap) high-lift system is not 
required. They conducted their experiment in the closed NASA Ames 7 by 10 foot wind tunnel and considered two test model configurations. The first one consisted of a NACA 632-215 Mod B main airfoil with a 30\% chord, halfspan hinged flap. In the second configuration, a CML segment replaced $29 \%$ of the flap span and provided a smooth transition from the flap to the adjacent main airfoil. A phased microphone array recessed in the wind tunnel wall on the model pressure side was used to perform the acoustic measurements. The freestream Mach number was 0.22 and results were reported for a $35^{\circ}$ flap deflection. Noise spectra were determined using conventional array beamforming methodology. The spectrum for the hinged flap configuration was found to be 6 to $8 \mathrm{~dB}$ quieter than that of an equivalent slotted Fowler flap configuration (the lower noise levels for the hinged flap were attributed to lower flap loading and a weaker side edge vortex system). The use of the CML segment reduced the apparent noise radiating from the side edge of the hinged flap; however, the noise associated with the CML hinged flap could not be quantified as it appeared to be lower than the wind tunnel background noise level.

Streett et al. ${ }^{22}$ tested the CML concept on a high-lift system configuration traditionally used on commercial transports. The test model consisted of a swept wing with a full-span leading-edge slat and a part-span Fowler flap. As was the case in Storms et al. ${ }^{23}$, the model was installed in a closed wind tunnel with a phased microphone array mounted along one of the test section walls, on the pressure side of the model. The freestream flow Mach number was 0.2 . Traditional beamforming techniques were used to process the acoustic data. The results were determined by extracting the peak levels of the beamform output. They indicated that a large reduction of the noise radiating from the flap side-edge region was achieved when compared to that obtained from a baseline (no CML) flap configuration. The noise reduction level was not quantified as the noise associated with the CML flap was below the perceived wind tunnel background noise level.

In the present study, acoustic and unsteady surface pressure measurements were performed on a CML flap model configuration in order to identify the noise source regions, quantify their strength and determine the directivity of the radiated noise. The acoustic measurements were performed in an open anechoic jet facility An outof-the-flow phased microphone array was used to acquire the data and the Deconvolution Approach for the Mapping of Acoustic Sources ${ }^{25}$ (DAMAS) method was applied to quantify the position and strength of the noise sources. The DAMAS method was chosen to analyze the acoustic data as it leads to a more accurate presentation of the noise source distribution than that obtained from traditional beamforming techniques. DAMAS was also applied to acoustic data that was acquired for a blunt side edge flap in a previous experiment ${ }^{13}$. The results obtained from the CML and blunt flap configurations are compared to quantify the levels of noise reduction. Finally, a Coherent Output Power (COP) method ${ }^{13}$, employing unsteady surface pressure and array acoustic measurements, is used to obtain a measure of the apparent noise source distribution in the trailing-edge (TE) region of the CML.

\section{A. Test Apparatus}

\section{Test description}

The CML flap experiment was performed at NASA Langley Research Center in the Quiet Flow Facility (QFF). The QFF is an anechoic, open jet facility equipped with a 2 by 3 foot rectangular open jet nozzle. The test model was supported above the nozzle by two vertical side plates that are mounted to the short sides of the nozzle. The test model (shown in Fig. 3) is a NACA $63_{2}-215$ main element airfoil with a half-span CML flap. The main airfoil TE thickness is 0.026 " ( \pm 0.002 ") in the region of the flap span and 0.016 " ( \pm 0.004 ”) over the rest of the span. The chord and span of the main airfoil are 16 and 36 inches, respectively. The main airfoil was positioned in the test section at an angle of attack of $16^{\circ}$ (as done in the flap noise experiment reported in Ref. 13, such that the local aerodynamics would properly represent high-lift device conditions on the flap and in the main element aft region). The CML flap consists of a Fowler flap with the mid-span side edge faired into the trailing edge of the main airfoil. As sketched in Fig. 4, the Fowler flap extends over a 15.75" span, followed by the CML transition which extends over a span of 4.5”. The chord of the Fowler flap and CML is 4.8 inches.

Two CML flap models were tested. The first one corresponds to a flap deflection angle of $39^{\circ}$ (with respect to the chordline of the main airfoil). The second model was a flap deflected at $29^{\circ}$. Thirty-four (34) unsteady surface pressure sensors (Kulites) were installed along the trailing edge of the $29^{\circ}$ and $39^{\circ} \mathrm{CML}$ models (see Fig. 4). The sensors were distributed along the suction side and pressure side of the models. The spanwise locations of the suction side sensors were within 0.125 " of the spanwise locations of the pressure side sensors. Following the TE contour line, the spacing between adjacent sensors was uniform (and equal to 0.5 ”) and the shortest distance from each sensor to the TE was $0.3^{\prime}$. The TE thickness of the $39^{\circ}$ CML flap was approximately $0.022^{\prime \prime}$ over the span of the Fowler flap and 0.016 for the CML, while for the $29^{\circ}$ CML flap, the TE thickness was approximately 0.019" over the span of the Fowler flap and 0.011 for the CML. 


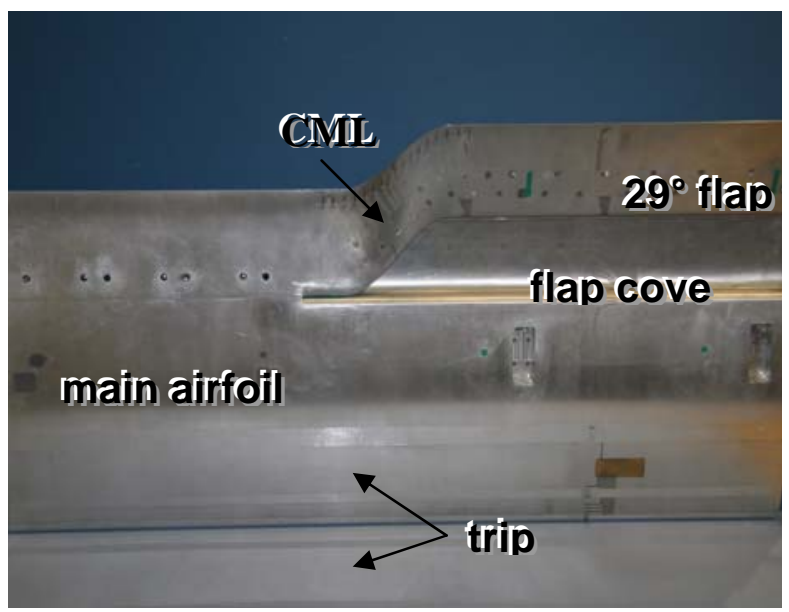

Figure 3. Main airfoil with $29^{\circ} \mathrm{CML}$ flap. View of test model pressure side.
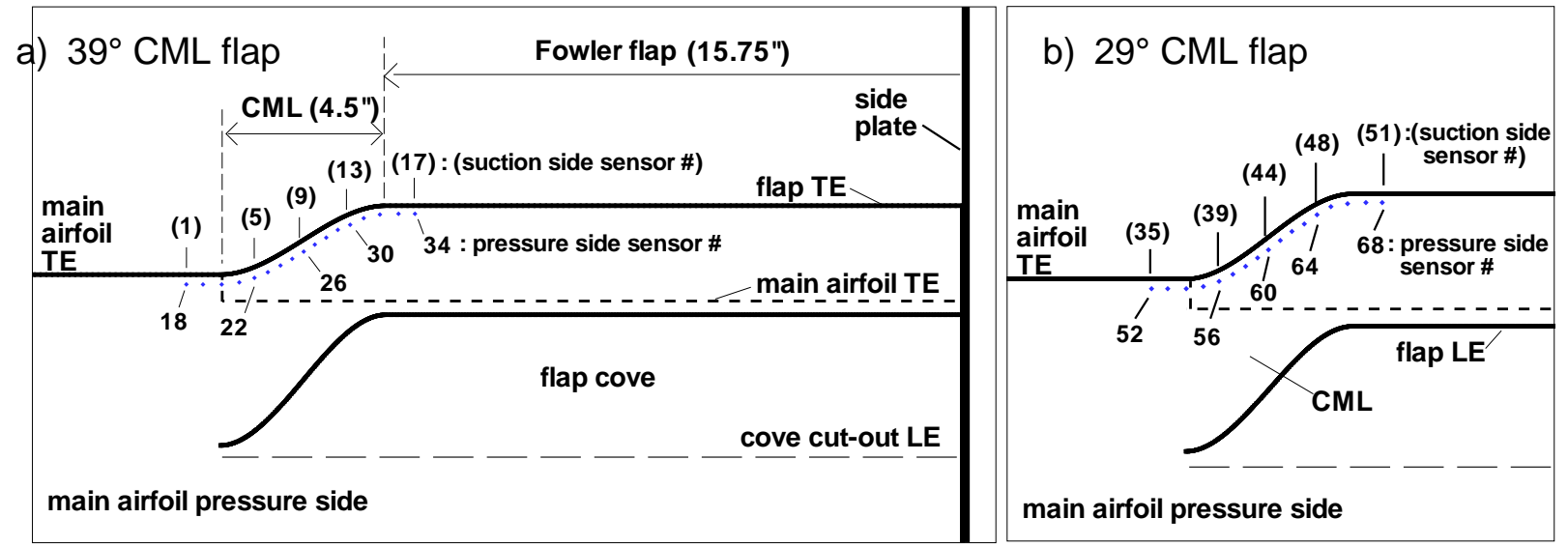

Figure 4. Unsteady surface pressure sensors distribution.

Strips of serrated tape (0.004" thick) were placed along the span of the main airfoil to trip the boundary layer and simulate turbulent boundary layer separation, as shown in Fig. 3. The strips were positioned along the model pressure and suction side at $9 \%$ chord, along the model pressure side at $27 \%$ chord, and along the airfoil leading edge (LE). For some of the configuration tested, 0.008" thick serrated tape was also placed along a portion of the main airfoil TE (see Fig. 5) in an attempt to control the noise that is seen in the flap cove region. The crests of the serrations were aligned with the airfoil TE, while the peaks extended 0.5” past the TE. The role of this TE modification is further discussed in this paper.

Langley’s Medium Aperture Directional Array, MADA ${ }^{26}$, consisting of 41 B\&K 1/8” microphones projecting from an acoustically treated frame, was used to acquire the acoustic data. The MADA microphones were arranged in 5 concentric irregular rings of 8 microphones each, with one microphone at the center of the rings. The array was positioned 5 feet from the model main element TE, in the mid-span plane. It was mounted on a pivotal boom and could be positioned around the test model at different elevation angles (the axis of rotation of the boom was aligned with the main airfoil TE). A sketch of the acoustic test set up is shown in Figure 6. A screen of thin fabric was installed over the face of the array to prevent wind gusts from increasing microphone self-noise.

Noise and surface pressure measurements were performed for the $39^{\circ}$ and $29^{\circ}$ CML flap models (with and without the main airfoil TE modification) at flow speeds ranging from Mach 0.07 to 0.17 (corresponding to a main element Reynolds number ranging from about 0.6 to 1.6 million) and with the MADA positioned at elevation angles ranging from $73^{\circ}$ to $141^{\circ}$. 


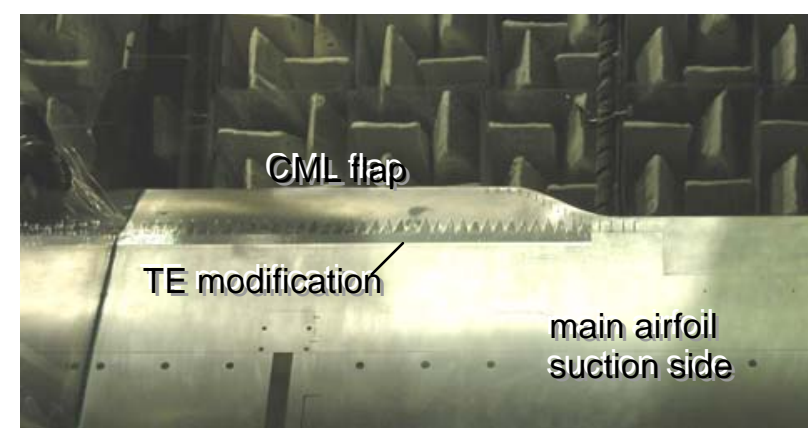

Figure 5. CML flap test model with main element TE modification.

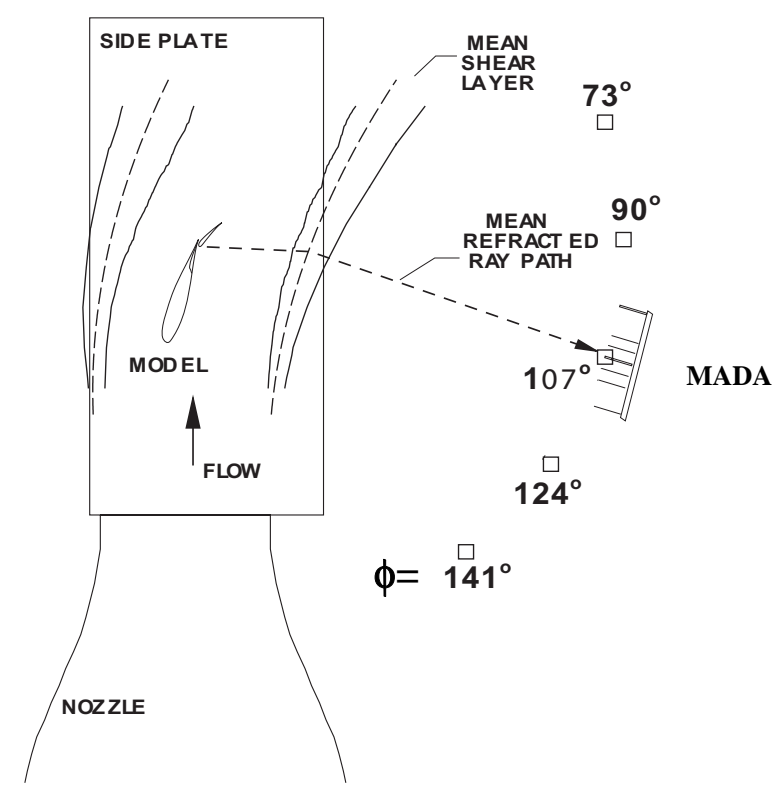

Figure 6. Sketch of test set up with phased microphone array measurement locations.

\section{B. Data Acquisition and Post-Processing}

Transient data recorders controlled by a workstation were used to acquire the data, simultaneously, from the 41 microphone channels and from the 34 pressure sensors channels at a sampling rate of $142.857 \mathrm{kHz}$. A low pass filter set at $50 \mathrm{kHz}$ was used to condition the outputs from each microphone and pressure sensor channel. The microphone signals were also high pass filtered at $300 \mathrm{~Hz}$. A cross-spectral matrix was constructed for each set of data acquired from the 34 pressure sensors and the MADA 41 microphone channels. Individual elements of the cross-spectral matrices were computed by partitioning each time signal into non-overlapping segments of $2^{13}$ samples. Each time history segment was then Fourier transformed using a Hamming window for signal conditioning. The resulting frequency resolution was $17.42 \mathrm{~Hz}$. This cross-spectral representation of the acquired acoustic and surface pressure data is used subsequently in the DAMAS and COP analysis of the data.

The DAMAS method was used to process the acoustic data to obtain spatial mapping of the noise sources. The first step of the DAMAS analysis consists of the application of standard diagonal removal beamforming ${ }^{25}$. The beamforming process is used to electronically "steer" the MADA over chosen locations about the test model. In the present analysis, the scanning plane consists of a 52" high by 50 " wide planar grid positioned through the main 
airfoil TE, parallel to the face of the array. The spacing used between grid points is 1 ”. The array output power spectrum for a chosen grid point, or steering location, is obtained from

$$
Y(\hat{\mathrm{e}})=\frac{\hat{\mathrm{e}}^{\mathrm{T}} \hat{\mathrm{W}}\left(\hat{\mathrm{G}}_{\text {array }}\right)_{\operatorname{diag}=0} \hat{\mathrm{W}}^{\mathrm{T}} \hat{\mathrm{e}}}{\left(\sum_{m=1}^{m_{0}} w_{m}\right)^{2}-\left(\sum_{m=1}^{m_{0}} w_{m}\right)}
$$

Where $\hat{\mathrm{e}}$ is the steering matrix (between the microphones and the steering locations), the superscript $\mathrm{T}$ indicates a complex transpose, $m_{0}$ is the number of microphones in the array, $\hat{G}_{\text {array }}$ is the cross-spectral matrix constructed from the data acquired from the $m_{0}$ microphone channels, and $\hat{W}$ is a frequency-dependent weighting-function row matrix containing $w_{m}$ weighting coefficients. $\hat{\mathrm{W}}$ is defined such that the array beamwidth remains approximately invariant (and about equal to 1 foot) in the frequency range between 5 and $40 \mathrm{kHz}$. In Eq.(1), the diagonal terms of the cross spectral matrices $\hat{G}_{\text {array }}$ are set to zero to help remove any potential microphone self-noise contamination ${ }^{26}$. In the next step of the DAMAS processing, a deconvolution algorithm is used to remove the effects of the array beamform response functions (which depend on array geometry, size, and frequency) from the beamformed output obtained in step one. This process involves solving the inverse problem of a linear system of equations,

$$
\hat{A} \hat{X}=\hat{Y}
$$

that relates the spatial field of grid point locations with beamformed outputs, $\hat{Y}$ (Eq. (1)), to equivalent acoustic source distributions, $\hat{X}$, at the same point locations. The resulting deconvolved array output presentation is explicit with the noise radiated from a region of interest determinable by a simple summation of the mean-squared pressures over that region.

A Coherent Output Power (COP) method $^{13}$ is used to obtain a measure of the noise source distribution along the span of the CML, near the TE. This method relates the output of the unsteady surface pressure sensors to the output of the MADA. When the MADA is steered to a surface pressure sensor location, the spectrum of the surface pressure sensor output that is coherent with the microphone array output can be obtained from ${ }^{29}$

$$
\operatorname{COP}_{S}=\frac{\left|G_{a, s}\right|^{2}}{G_{a}}=\gamma_{a, s}^{2} G_{s} .
$$

$C O P_{S}$ is the coherent output power spectrum, $G_{s}$ is the pressure sensor auto-spectrum, $G_{a}=Y(\hat{e})$ is the autospectrum obtained from the output of the array when beamformed to the pressure sensor location, $G_{a, s}$ is the crossspectrum between the array and pressure sensor outputs, and $\gamma_{a, s}^{2}$ is the coherence. For this calculation, the array microphone time signals are offset by an amount $\tau_{a, s}$, which should equal the time required for the noise to travel from the pressure sensor, through the shear layer, to the center of the array. For the present data, a block time offset (close to $\tau_{a, s}$ ) between the microphone and surface sensor signals was applied prior to the determination of $G_{a, s}$. Final adjustments of $\tau_{a, s}$ for $G_{a, s}$ are done in the frequency domain. As explained in Ref. 13, this time shifting procedure in done to avoid bias and statistical errors. It also effectively removes the phase that is related to the noise transmission time, from the $G_{a, s}$ (and $C O P_{S}$ ) cross-spectra. The application of the COP method to the CML flap test data is described in the following section. 


\section{Results and Discussion}

The spectra and directivity determined for the noise sources for the CML flap configurations are presented here. Also, noise results are compared to those of a blunt flap configuration.

The data presented for the blunt flap were acquired in a previous QFF experiment ${ }^{13}$. The main element and Fowler flap configuration from the previous experiment matches that of the present CML configuration, except for the presence of a simple blunt side edge and the presence of support brackets. A picture of the blunt flap test model is shown below in Fig. 7. The flap was attached to the main airfoil element with a set of brackets that were intended to have minimal interference with the flow field. Initially, in the present experiment, these brackets were also mounted to the CML flap model at the same spanwise and chordwise locations to evaluate the noise contribution of the brackets. However, the brackets were found to have insignificant impact on the acoustic (and surface pressure) measurements, so the results obtained for the CML flap without brackets are used in the present analysis. Structurally, the CML flap did not require the brackets.

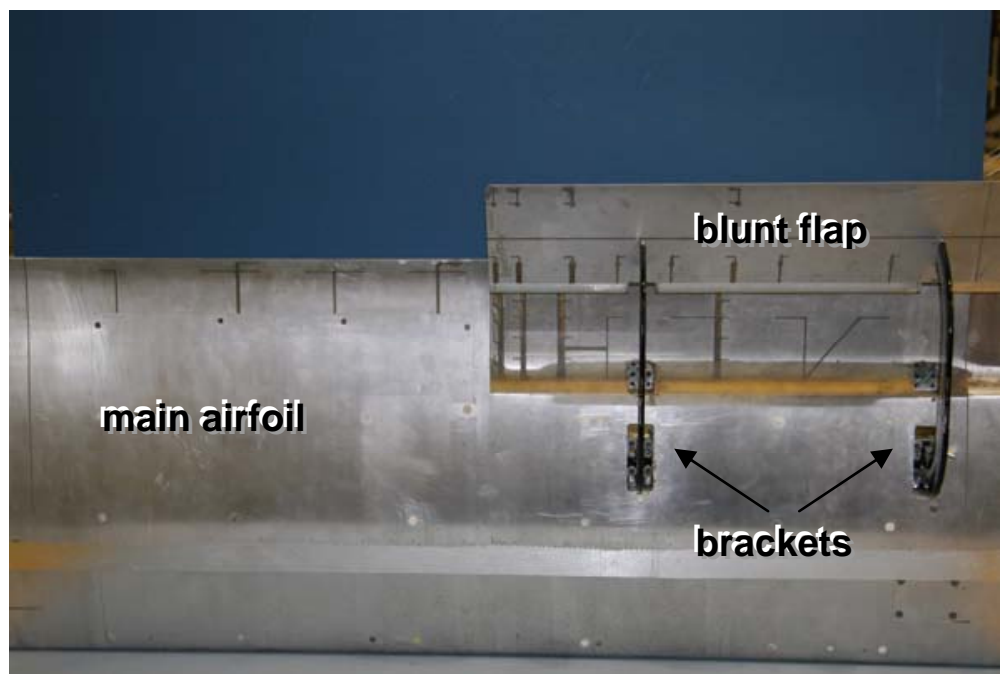

Figure 7. Main airfoil with $29^{\circ}$ blunt half-span flap. View of test model pressure side.

The test configurations used in the CML and blunt flap experiments were the same, with the exception of the phased array used to acquire the acoustic data. The Small Aperture Directional Array, SADA ${ }^{28}$, was employed for the blunt flap. The SADA corresponds to the inner 4 rings of microphones and the single central microphone of the MADA. The SADA is designed to maintain a nominal 1-foot beamwidth between 10 and $40 \mathrm{kHz}$ versus the 5 to 40 $\mathrm{kHz}$ range for the MADA. Hence, between 10 and $40 \mathrm{kHz}$, the SADA and MADA have identical characteristics, while at lower frequencies, the noise measurements performed with the SADA have reduced resolution (broader beamwidth). This correspondingly reduces the related DAMAS resolution.

\section{A. Noise Source Distribution in the CML Trailing Edge Region}

The COP processing, employing Eq. (3), is used to identify noise source characteristics in the TE region of the CML. This was also done for the blunt flap configuration in Ref. 13. There, the sensors were located in the strong noise-producing region .06 inch from the blunt edge. It was found that for both blunt flap angle settings tested, the strongest noise strengths were near $65 \%$ chord on the suction side and near $50 \%$ chord on the pressure side of the flap edge. Corresponding information was desired for the CML. Sensors were placed as close as possible, at .3 inch, from the relatively sharp CML trailing edge. Because the sensors had to be placed somewhat away from the edge, their outputs may be more influenced by indirectly coherent sources rather than just the noise-producing scatter found for the blunt edge sensors. Hydrodynamic convection effects (part of which may be due to convectingturbulence on its way to radiate as noise) and noise radiating along the surface (from other sources) can significantly contribute to the measured pressure levels. The COP method extracts the portion of this complex pressure field that correlates with the array measurements. Thus, in the present CML study, the COP may be expected to provide 
somewhat more indirect evidence of the noise source distribution than for sensors closer to the edge (as in the blunt side edge case of Ref. 13).

In this analysis, the MADA is positioned at $\phi=107^{\circ}$, approximately normal to the chordline of the main element. Figure 8 shows a set of COP processed spectral data for sensor \#7 (on the suction side, away from the array) and the array output when focused to this sensor. Shown are the auto-spectra $G_{a}$ and $G_{s}$, for the array and sensor, respectively, the cross-spectrum $G_{a, s}$, the coherence $\gamma_{a, s}^{2}$, as well as the cross-spectral phase $\left(\varphi_{a, s}-\omega \tau_{a, s}\right)$ associated with the $C O P_{s}$. It is seen in this figure that $\gamma_{a, s}^{2}$ is greatest at frequencies below about 3.5 $\mathrm{kHz}$, and that the phase relation between the MADA and pressure sensor outputs breaks down above $3.5 \mathrm{kHz}$. Between $1.0 \mathrm{kHz}$ and $3.5 \mathrm{kHz}$, the phase approaches zero. Below about $1.0 \mathrm{kHz}$, the phase relation changes, indicating (for that frequency range) the presence of noise sources located elsewhere. The fact that the phase is seen to stay near zero in the coherent range can be significant. The $\tau_{a, s}$ employed corresponds to the time required for radiation from the TE plus an additional time increment to account for the distance between the sensor and the TE. The phase behavior is consistent with either the pressure field of turbulence or sound passing the sensor on its way to scatter from the trailing edge to the array (note that, because of the dipole nature of TE noise, a phase approaching $180^{\circ}$ would be consistent with a dominant contribution from sound traveling back to the sensor after being generated at the TE). A detailed analysis of the phase behavior as reported in Ref. 13 and 29 is beyond the scope of the present study whose main intent is to present some measure of the distribution of the apparent noise sources in the TE region of the CML.
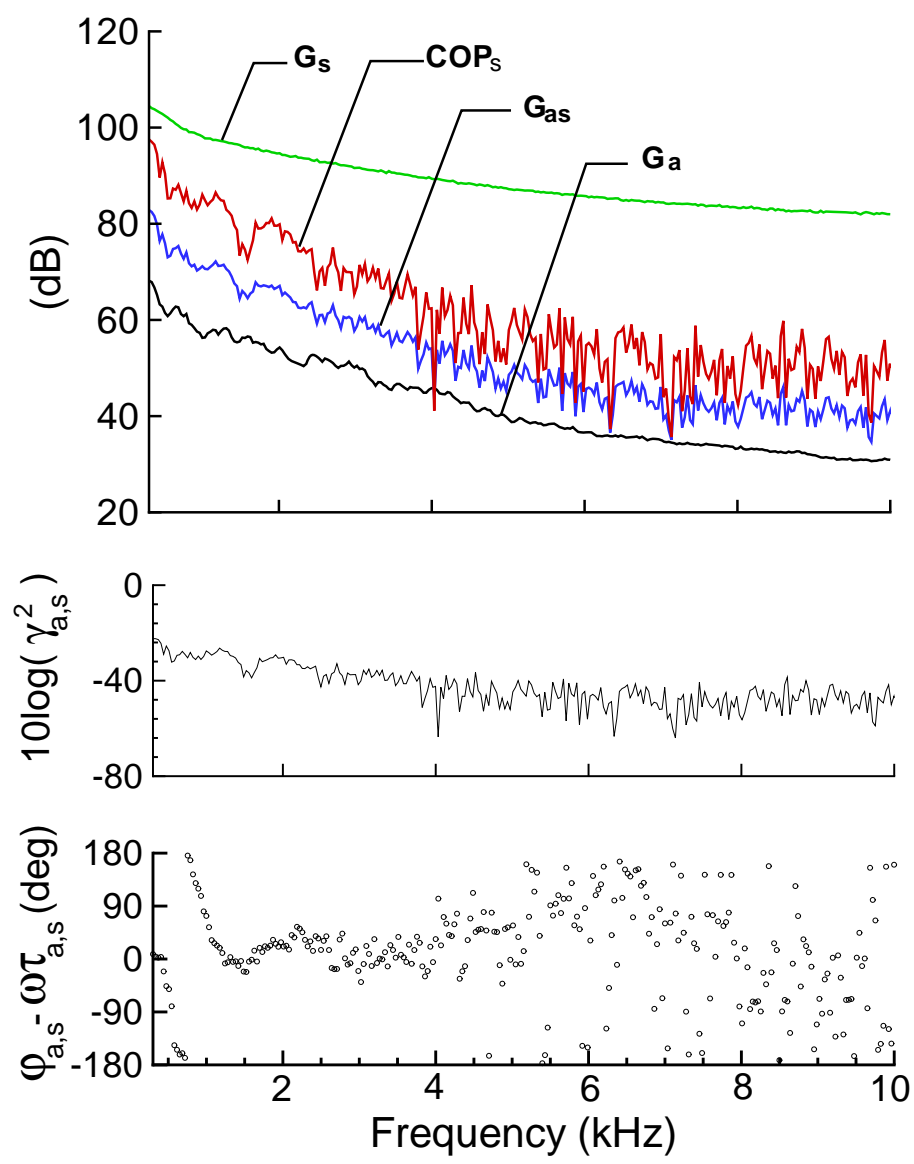

Figure 8. Coherent output power spectral processing results for the $39^{\circ} \mathrm{CML}$ flap model, relating sensor \# 7 pressure measurement and the MADA noise measurement for $M=0.17$ and $\phi=107^{\circ}$. The spectral resolution is $\Delta f=34.88 \mathrm{~Hz}$, but levels are referenced to $\Delta \mathrm{f}=1 \mathrm{~Hz}$. 
Figure 9 presents the spanwise distributions of integrated band-limited levels from both the surface sensor autospectra and $C O P_{S}$ spectra. The levels are shown for the sensors located along the pressure and suction sides of the CML for both the $29^{\circ}$ and $39^{\circ}$ flap angles, for a flow Mach number of 0.17 . The levels result from integrating the auto-spectra $\left(G_{s}\right)$ for each sensor from 1.0 to $3.5 \mathrm{kHz}$. The corresponding integrated $\mathrm{COP}_{S}$ levels are determined by pressure-squared and phase summing (vectorial summation) after the previously-discussed phase adjustment for $\tau_{a, s}$.

Figure 9 is used to locate the apparent source distributions for noise in the 1.0 to $3.5 \mathrm{kHz}$ frequency range for the CML. Note in Fig.9 that the locations of the start and end of the CML link are shown with respect to the sensor locations. So-called $0 \%$ and 100\% CML length locations (which correspond, respectively, to the $43.8 \%$ and 55.8\% model span locations) are indicated near sensors 20 and 32 for the $39^{\circ}$ flap, and near sensors 54 and 66 for the $29^{\circ}$ flap. The results indicate that the noise is distributed, but most strongly radiates in somewhat different regions for the CML pressure and suctions sides. For the $39^{\circ}$ flap, the strength of the apparent noise sources peaks around $30 \%$ of the CML length on the suction side and around $50 \%$ on the pressure side. For the $29^{\circ}$ flap, the strength peaks closer to the flap TE and over a broader portion of the CML length (between $25 \%$ and $70 \%$ on the suction side and around $70 \%$ on the pressure side). The corresponding distributions obtained for a flow Mach number of 0.11 (not shown), were found to have the same basic characteristics as for the 0.17 Mach number test condition although the coherence was somewhat lower. Note again that the sources present below $1 \mathrm{kHz}$ are not addressed here. Above 3.5 $\mathrm{kHz}$, the COP results do not indicate strong trailing edge contributions to the radiated noise field. In the following section, this view is reinforced by the separate DAMAS noise distribution analysis.

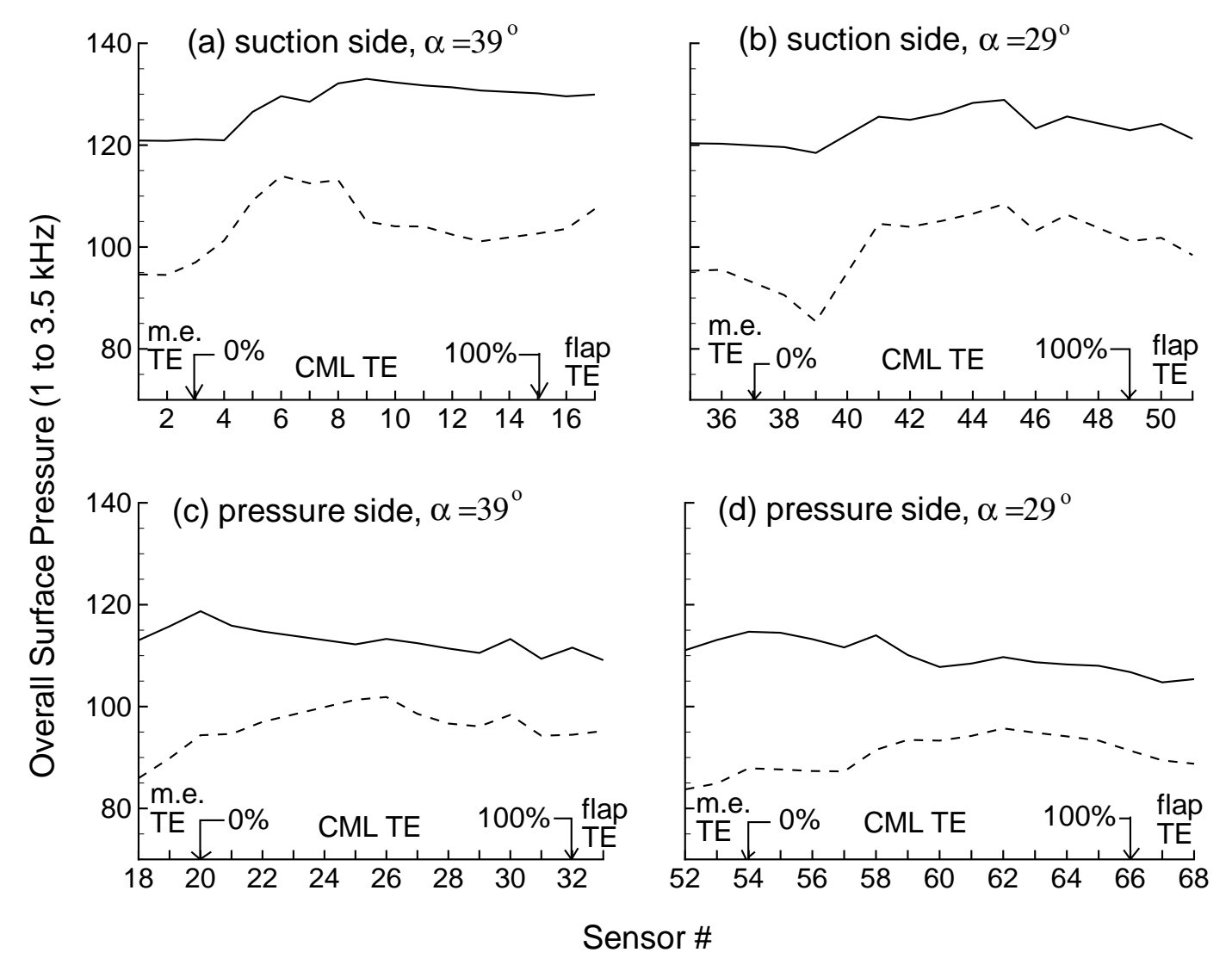

Figure 9. Distribution over the CML, of band limited (1 to $3.5 \mathrm{kHz}$ ) overall surface pressure levels and related COPs levels. $\phi=107^{\circ}$ and $M=0.17$. The keys for $\mathrm{dB}$ levels are as follows: - pressure sensor integrated auto-spectra; - - - integrated COPs spectra. The main element (m.e.), CML and flap TE locations are indicated. 


\section{B. DAMAS Noise Source Distribution}

DAMAS processing of the phased microphone array data was performed to obtain spatial distributions of the noise sources for the CML flap, for the blunt flap, and for the CML flap with the main element TE modification described earlier. The DAMAS noise source distributions obtained at a flow Mach number of 0.17 , for the $39^{\circ}$ flap configurations, are presented in Fig. 10. Results are shown for sets of bands that sum four standard one-third-octave frequency bands. For these measurements, the phased array was located at $\phi=107^{\circ}$ (as in the COP analysis). Spanwise and chordwise coordinates shown are with respect to a 52" high and 50" wide planar grid (scanning grid used for the DAMAS processing) positioned through the main element TE, parallel to the face of the array. This places the grid approximately along the chordline of the main airfoil when $\phi=107^{\circ}$. The profile of the test model shown is as seen by the center of the phased array. The results presented were obtained by first generating DAMAS maps for each narrowband frequency. The narrowband results were then summed (on a pressure-squared basis) to obtain one-third octave band maps from which four bands were themselves summed to obtain each data frame shown in Fig. 10. The center frequencies of the first and last one-third octave bands included in each sum are indicated.

It is seen that for the $39^{\circ} \mathrm{CML}$ flap, at the lower frequencies, the noise radiates most strongly from the TE region of the CML as well as from the LE region of the Fowler portion of the flap. The latter observation is consistent with the results of the COP analysis which indicated that noise radiates from the CML TE region, between approximately 1 and $3.5 \mathrm{kHz}$. It is also seen in Fig.10 that at slightly higher frequencies, the dominant noise sources become more concentrated near the LE of the CML and along the flap LE and cove regions. Above the 6.3 $\mathrm{kHz}$ band, the DAMAS maps reveal distributions of weaker noise sources. Between 8 and $16 \mathrm{kHz}$, the strongest noise sources are located near the LE of the CML, while from 20 to $40 \mathrm{kHz}$, the dominant noise sources appear located in the flap cove region of the main element. The DAMAS noise source distributions in the $20 \mathrm{to} 40 \mathrm{kHz}$ range obtained for the $39^{\circ} \mathrm{CML}$ flap with the TE mod show that the treatment weakened the sources located in the flap cove region. However, it also appears to have led to a slight increase in the strength of the noise sources located in the CML region, for lower bands.

For the corresponding blunt flap configuration, the DAMAS noise source distributions indicate that the strongest noise sources are concentrated in the side edge region of the flap. This is clearly revealed in the DAMAS results obtained above $6.3 \mathrm{kHz}$. At lower frequencies, the flap cove appears as an additional region of noise emission. However, these frequencies are outside the SADA design range. At $6.3 \mathrm{kHz}$ the SADA beamwidth is approximately 1.5', but it increases to 3' (the full span of the model) at $3.15 \mathrm{kHz}$. Hence, at these frequencies, DAMAS has difficulty producing accurate and spatially discriminating results. It is seen that DAMAS could not quite resolve the noise source distributions below the $3.15 \mathrm{kHz}$ band. Thus, referring to Fig. 10, the array resolution for the measurements acquired with the SADA is deemed marginal in the 3.15 to $6.3 \mathrm{kHz}$ band range, and poor for the lowest band. For the measurements obtained with the MADA, the resolution becomes marginal for the data acquired in the 1.25 to $2.5 \mathrm{kHz}$ band range. Note that a similar distribution of the noise sources presented in Fig. 10 was observed (results not shown) for the $39^{\circ}$ flap deflection for Mach number 0.11 , and for the $29^{\circ}$ flap deflection for Mach numbers 0.17 and 0.11 .

The presence of apparent noise emission, seen in the region where the flap is mounted to the side plate, is due to noise reflected from the model region. Because of likely partial coherence with model noise, this may slightly affect the results obtained near the side plate, since DAMAS is based on the assumption of uncorrelated source distributions. DAMAS- $\mathrm{C}^{30}$ (which extends DAMAS to allow for both coherent and incoherent noise sources) could have been applied to resolve the noise sources distribution in the side plate region somewhat more accurately, but this was not deemed necessary in the scope of the present study.

\section{CML and Blunt Flap Noise Spectra}

One-third octave band spectra of the noise radiating from different regions of the CML and blunt flap models are presented in this section. These spectra are obtained from the DAMAS maps generated for each one-third octave band. The spectral level for a given one-third octave frequency band is obtained by simply summing the mean squared pressures over the region of interest. In Fig. 11(a), the following zones are defined: zone A which encloses the TE region of the CML, zone B which encloses most of the LE region of the CML (also of the narrow gap between the CML suction side and the main airfoil pressure side), zone $\mathrm{C}$ which encloses the flap and cove regions, and zone D which encloses reflections from zone C. These zones are defined in the main airfoil chordline plan, in terms of tunnel coordinates (in Fig. 11, they are shown projected along the DAMAS scanning grid). It is seen in Fig. 
11(b) that for the blunt flap configuration, the same zone A encloses the side edge of the flap, while zone B encloses the part of the cove region that is closest to the side edge.

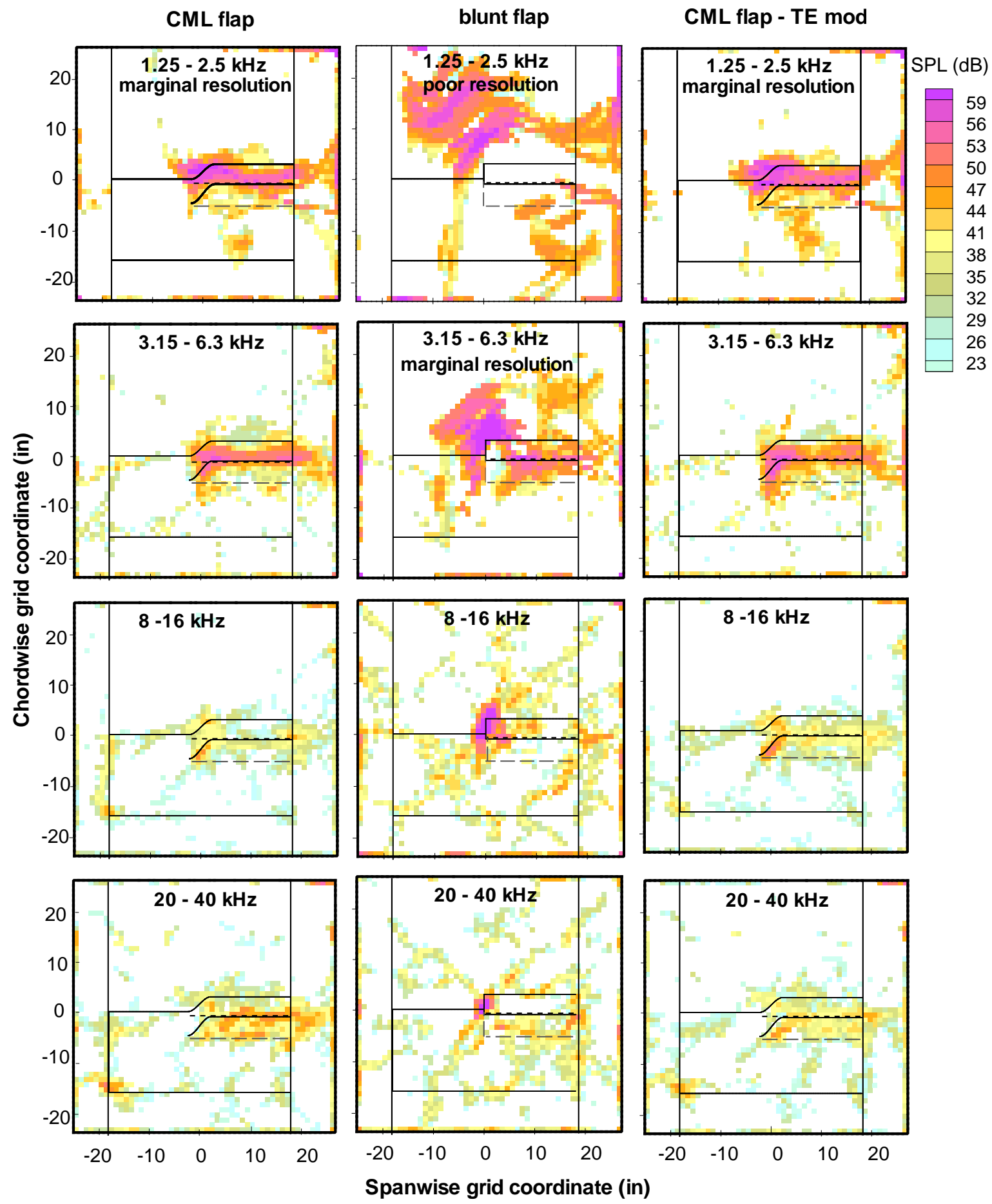

Figure 10. DAMAS noise source distributions in summed one-third octave bands for the $39^{\circ} \mathrm{CML}$ and baseline flap test models. The flow Mach number is 0.17. The range of one-third octave band center frequencies are indicated. 
a) $39^{\circ} \mathrm{CML}$ flap

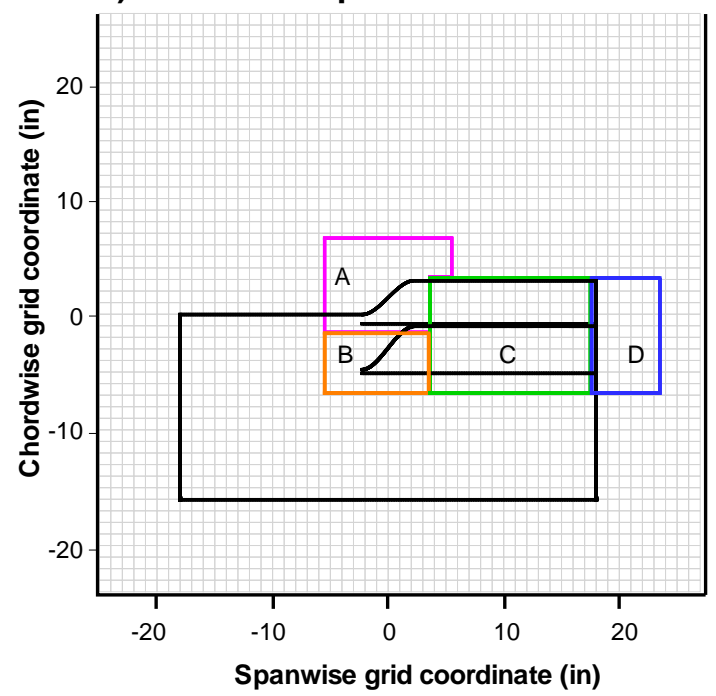

c) $39^{\circ} \mathrm{CML}$ flap

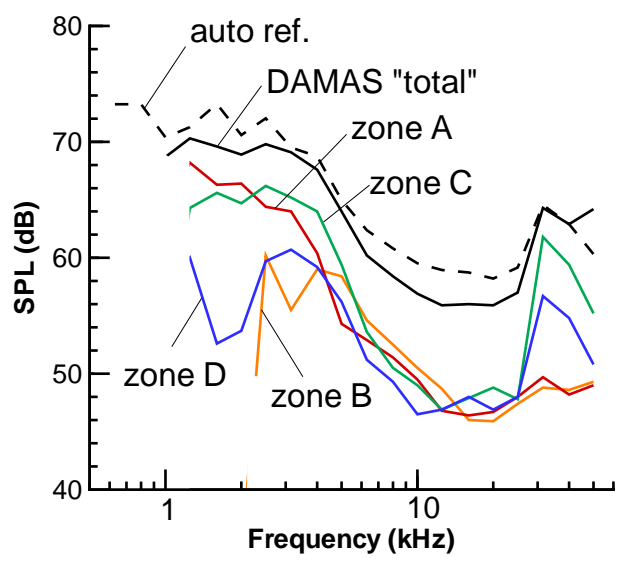

b) $39^{\circ}$ blunt flap

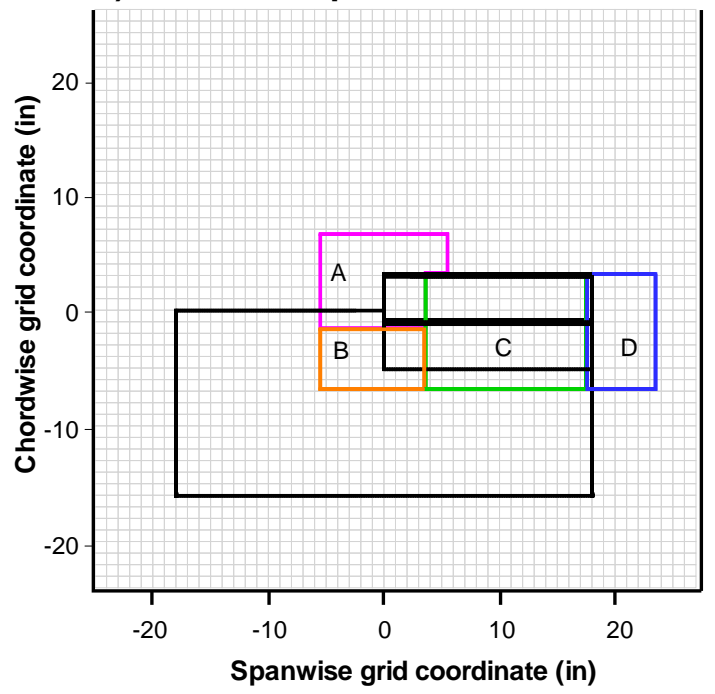

d) $39^{\circ}$ blunt flap

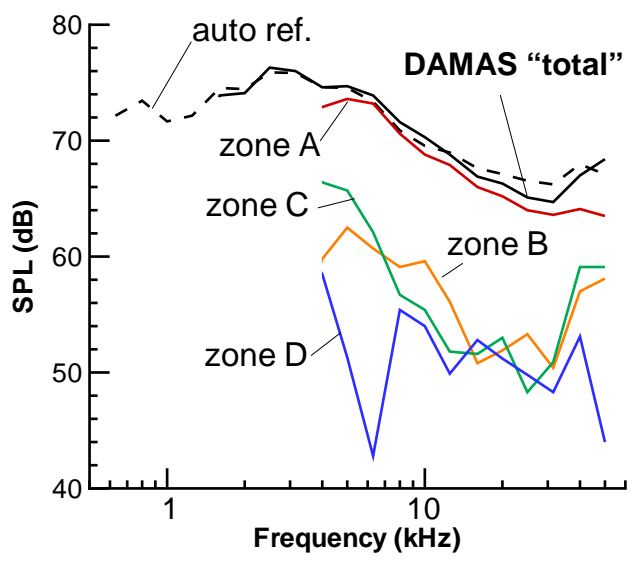

Figure 11. Integration area definition in DAMAS scanning grid for $\phi=107^{\circ}$ :

a) $39^{\circ} \mathrm{CML}$ flap, b) $39^{\circ}$ blunt flap. One-third-octave band spectra for 0.17 Mach number and $\phi=107^{\circ}$ : c) $39^{\circ}$ CML flap, d) $39^{\circ}$ blunt flap.

The one-third octave band spectra obtained for each zone are presented in Fig. 11(c) for the $39^{\circ} \mathrm{CML}$ flap, and in Fig. 11(d) for the blunt flap. The flow Mach number is 0.17 and the phased array (MADA for the CML flap, SADA for the blunt flap) is located at $\phi=107^{\circ}$. This position of the phased array corresponds to the model being in a flyover position. The autospectrum from an individual microphone of the array, as well as the "total" DAMAS spectrum are also shown in these figures for reference. The levels of the "total" DAMAS spectrum are determined by summing the mean squared pressures over the entire region covered by the scanning grid (borders excluded). Hence, the SPL levels in the "total" DAMAS spectrum should be close to those measured by the single microphone (or lower when the array discriminates against extraneous sources located outside of the scanning region). The zonal spectra (spectra obtained for zones A, B, C and D) are only shown down to $4 \mathrm{kHz}$ for the blunt flap, as the beamwidth of the SADA at lower frequencies is too broad to properly capture the noise radiating from each individual zone. Because of the better spatial resolution of the MADA in the low frequency range, the zonal spectra 
obtained for the CML flap are shown down to $1.25 \mathrm{kHz}$ (the SPL levels are expected to have little contamination from extraneous sources above that frequency).

It is seen in Fig. 11(d) for the blunt flap that the noise radiating from the side edge (zone A) clearly dominates the spectra for all frequencies, and that the single microphone and total DAMAS auto spectra are in agreement. The spectra for zones B, C, and D collapse above $10 \mathrm{kHz}$, but are still $15 \mathrm{~dB}$ below the levels for zone A. For the CML flap (Fig. 11(c)), the noise radiating from the CML TE (zone A) and the flap and cove regions (zone C) are the most significant contributors to the noise spectra, below approximately $4 \mathrm{kHz}$. It is also seen that a peak in the spectra for zone $\mathrm{C}$ occurs at higher frequencies for both the blunt and CML flap configurations. The cause of this spectral peak is discussed further below. The zonal spectra obtained for the $29^{\circ}$ flaps, or for lower Mach numbers, also displayed the same characteristics. In the remainder of this discussion, the total area enclosed by zones A and B will be referred to as the blunt-edge region for the blunt flap configuration, and as the CML-edge region for the CML flap configuration. Similarly, the area enclosed by zone $\mathrm{C}$ for the blunt and CML flap configurations, will be respectively referred to as the blunt-cove and CML-cove regions.

In Fig. 12, the spectra of the noise radiating from the CML-edge and CML-cove regions are compared to the spectra obtained for the blunt-edge and blunt-cove regions. Results are shown for Mach numbers 0.11 and 0.17 , and for the two flap deflection angles tested. Below $4 \mathrm{kHz}$, the blunt "total" DAMAS spectrum is displayed to give an approximate representation of the blunt-edge noise spectra in that lower frequency range (note that this approximation is very good if the flap side edge noise continues to dominate the spectra in that frequency range).

a) $39^{\circ}$ flap and Mach 0.11

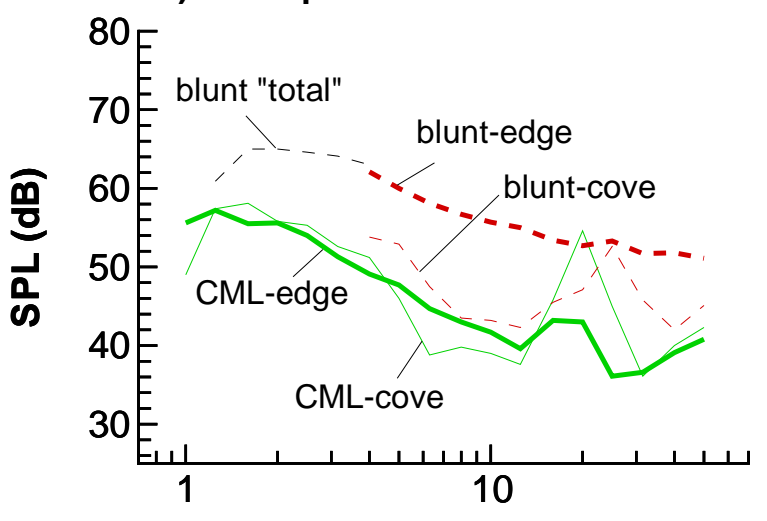

c) $29^{\circ}$ flap and Mach 0.11

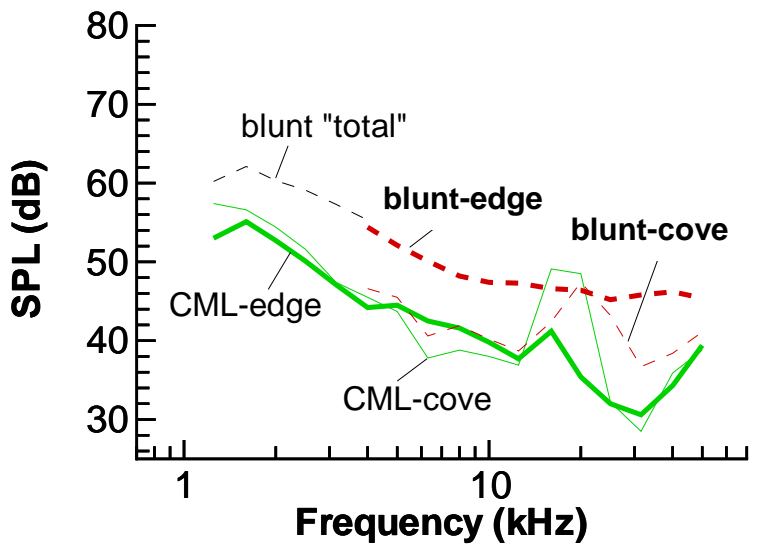

b) $39^{\circ}$ flap and Mach 0.17

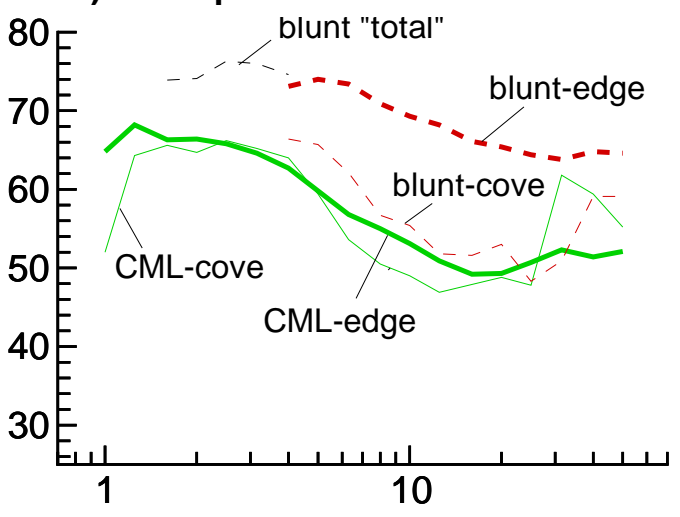

d) $29^{\circ}$ flap and Mach 0.17

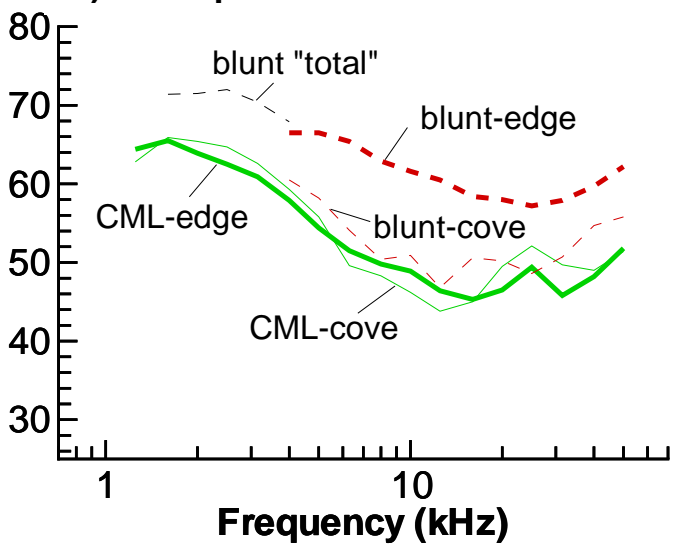

Figure 12. Edge and cove noise one-third-octave band spectra for the CML and blunt flaps. Phased array positioned at $\phi=107^{\circ}$. 
It is seen in Fig. 12 that the CML-edge spectra are 5 to $17 \mathrm{~dB}$ lower than the blunt-edge spectra. Higher levels of noise reduction are achieved for the $39^{\circ}$ flap than for the $29^{\circ}$ flap. The results also indicate that over most of the frequency range, the noise from the blunt flap side-edge is significantly louder (by about $10 \mathrm{~dB}$ ) than that from the cove region. For the CML flap, the noise from the CML and cove regions are close in level over most of the frequency range. A spectral peak in the cove noise spectra is present at high frequencies for both the CML and blunt flap configurations. The frequency of this peak increases with increasing Mach number (and appears to scale with the Strouhal number), and is lower for the $29^{\circ}$ compared to the $39^{\circ}$ case. The CML-cove and blunt-cove spectral peaks are close in amplitude, but the frequency of the peak is slightly higher for the blunt edge. At a Mach number of 0.11 , the peaks surpass the blunt-edge noise level. As will be shown next with the main airfoil TE modification, the spectral peak in the noise measured in the cove region of the flap is strongly related to noise that is scattered at the TE of the main airfoil and channeled into the flap cove region. The purpose of the main airfoil TE modification was to decrease the level of noise seen in the cove region by reducing the strength and coherence of the vorticity coming off the TE. The effect of the TE modification on the CML-cove noise spectral peak is shown in Fig. 13. The CML-edge and CML-cove spectra for the CML flap with and without TE modification are shown. It is seen that the TE modification was generally effective in reducing the large high-frequency CML-cove noise spectral peak. However, it also resulted in an increase of the CML-cove and CML-edge spectral levels at lower frequencies. The CML-cove and CML-edge spectral levels were found to be sensitive to very small geometric variations of the main airfoil TE treatment. There was no effort made to find a more optimal noise reduction treatment (modification). A study similar to that reported in Ref. 32 on slat cove noise, would be required to determine how the TE thickness, the gap (between the flap and the main element) and the local flow speed may affect the amplitude and frequency of the spectral peak. Nevertheless, the present results show the potential importance of main element TE geometry on the cove noise for both the blunt and the CML flaps. The cove noise levels shown are for a 14" span of the cove area. Assuming a spanwise distribution of uncorrelated noise sources, cove noise levels would increase by $3 \mathrm{~dB}$ for each doubling of the cove span.

a) $39^{\circ}$ flap and Mach 0.17

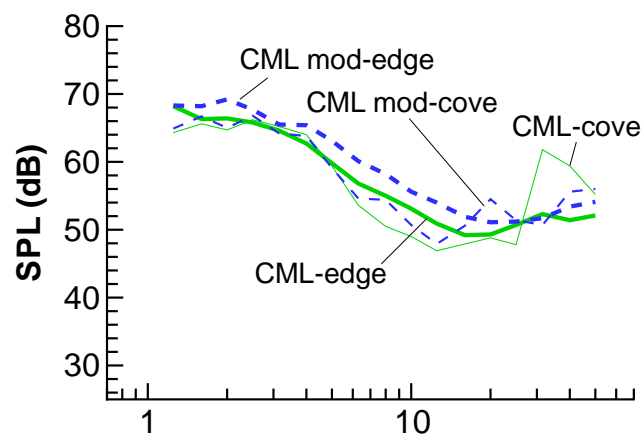

c) $29^{\circ}$ flap and Mach 0.17

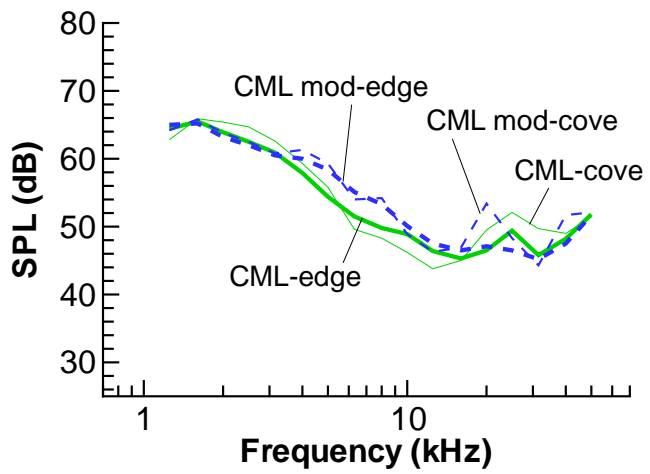

b) $39^{\circ}$ flap and Mach 0.11

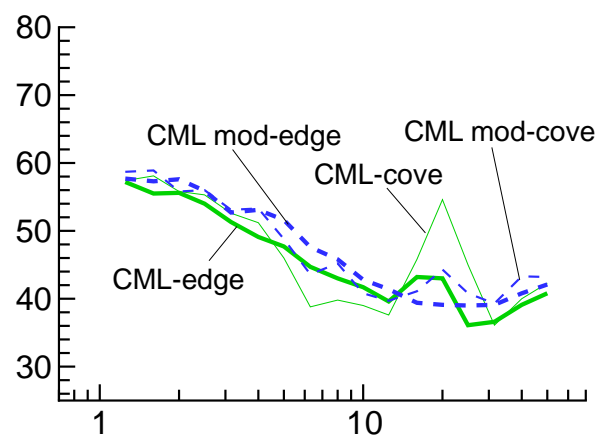

d) $29^{\circ}$ flap and Mach 0.11

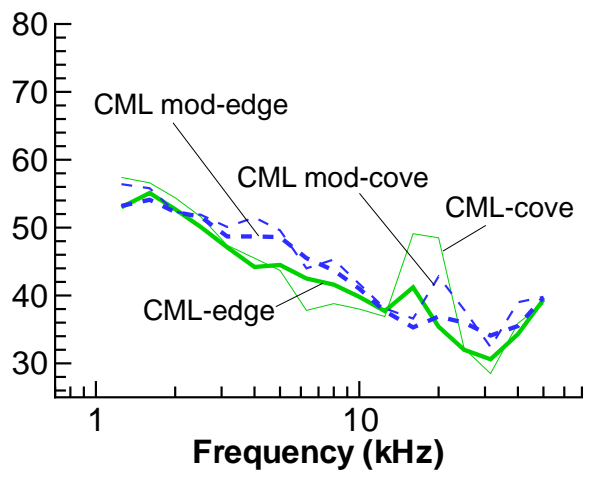

Figure 13. Edge and cove noise one-third-octave band spectra for the CML flap with TE modification and without (data same as in Fig. 12). Phased array positioned at $\phi=107^{\circ}$. 


\section{Spectral Directivity}

Figure 14 shows the directivity of the CML-edge and blunt-edge noise spectra for flyover angles (array mechanical elevation angles) $\phi=73^{\circ}, 90^{\circ}, 107^{\circ}, 124^{\circ}, 141^{\circ}$, for a flow Mach number of 0.17 . (Note that the corresponding emission (retarded) angles which are preferred when the data is used to develop a noise prediction model, can be derived in the manner shown in Ref. 33). In Fig. 14, it is seen that the blunt flap spectral levels are less sensitive to directivity angle variation than the CML flap. For the blunt flap, the spectra acquired at the different flyover angles are within $3 \mathrm{~dB}$ of each other over most of the frequency range, while for the CML flap, the difference in spectral levels between the most downstream spectrum $\left(\phi=73^{\circ}\right)$ and the most upstream spectrum $(\phi=$ $141^{\circ}$ ) is as much as $9 \mathrm{~dB}$. For both the CML-edge and blunt-edge noise spectra, the spread in the spectral levels broadens in the frequency range over which the cove noise spectral peak occurs. This suggests that the tonal spectral region boundaries crosse into both zone $\mathrm{A}+\mathrm{B}$ and zone $\mathrm{C}$.

Directivity spectra for CML-cove and blunt-cove noise are shown in Fig. 15. It is seen that the spectral peak present in the high frequency range maintains the same center frequency for the different directivity angles. The difference in peak levels between the most upstream and downstream directivity angles is as much as $15 \mathrm{~dB}$ for the $29^{\circ} \mathrm{CML}$ flap and $12 \mathrm{~dB}$ for the $39^{\circ} \mathrm{CML}$ flap. This difference in level is a little less pronounced for the blunt flap.

The CML-edge, CML-cove, blunt-edge and blunt-cove directivity spectra for Mach 0.11 (not shown) were found to have similar characteristics as those observed at Mach 0.17. The spectral levels for the lower speed were, however, uniformly lower by about $10 \mathrm{~dB}$ (except for the level of the CML-cove and blunt-cove spectral peaks which were only lower by about $5 \mathrm{~dB}$ ).

a) $39^{\circ} \mathrm{CML}$ flap

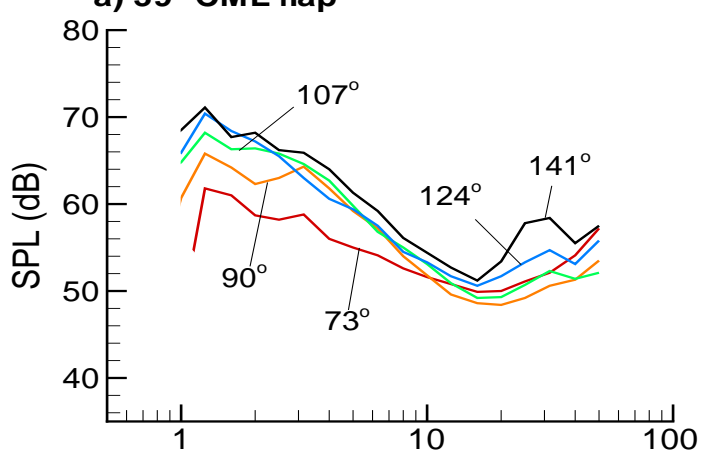

c) $29^{\circ} \mathrm{CML}$ flap

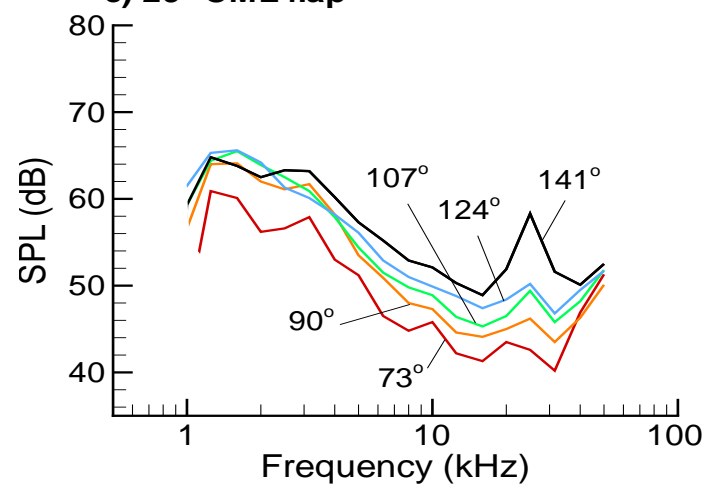

b) $39^{\circ}$ blunt flap

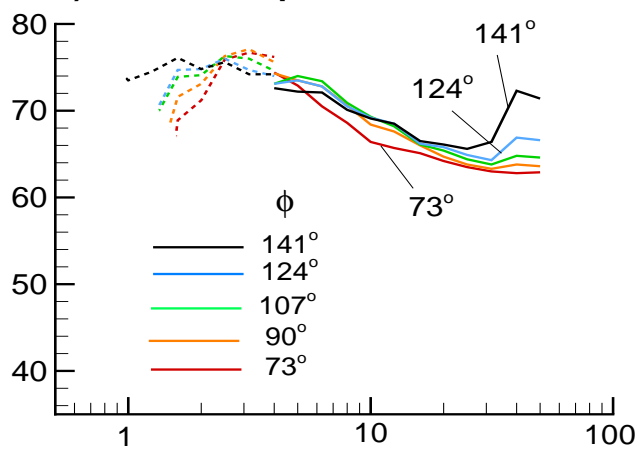

d) $29^{\circ}$ blunt flap

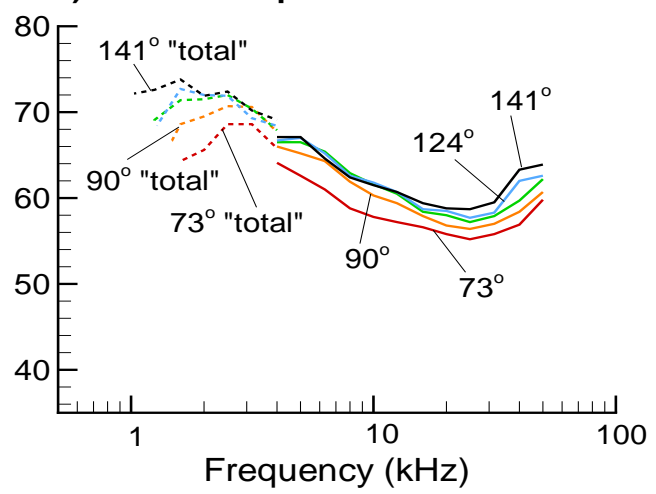

Figure 14. Edge-noise directivity one-third-octave band spectra for Mach 0.17. Solid lines: zone A+B, dashed lines: DAMAS “total”. 
a) $39^{\circ} \mathrm{CML}$ flap

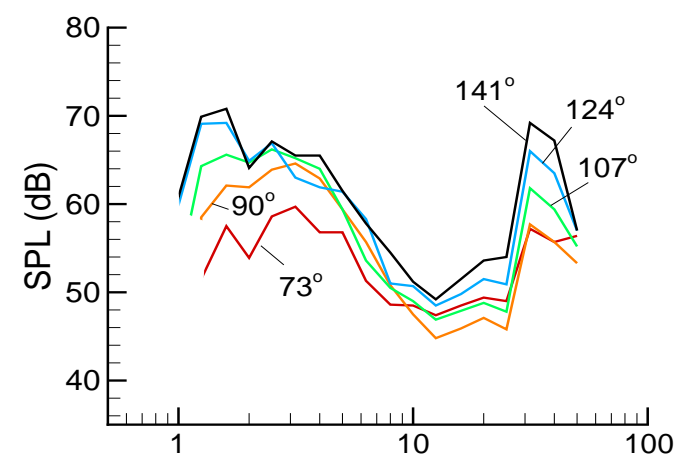

c) $29^{\circ} \mathrm{CML}$ flap

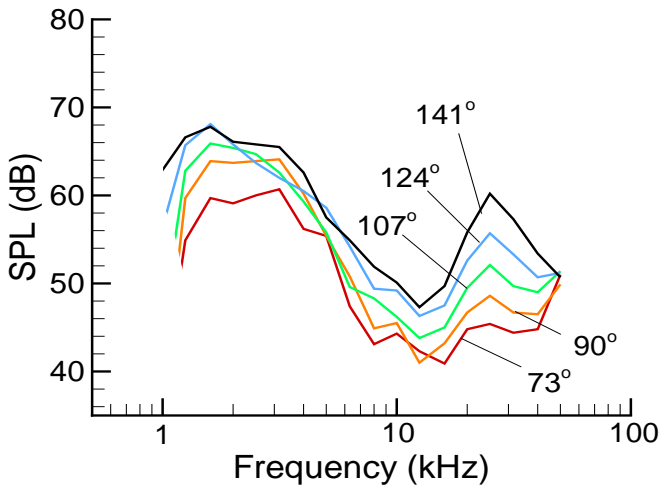

b) $39^{\circ}$ blunt flap

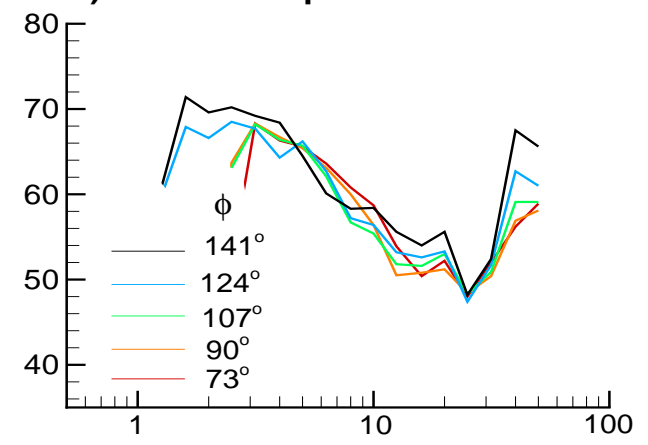

d) $39^{\circ}$ blunt flap

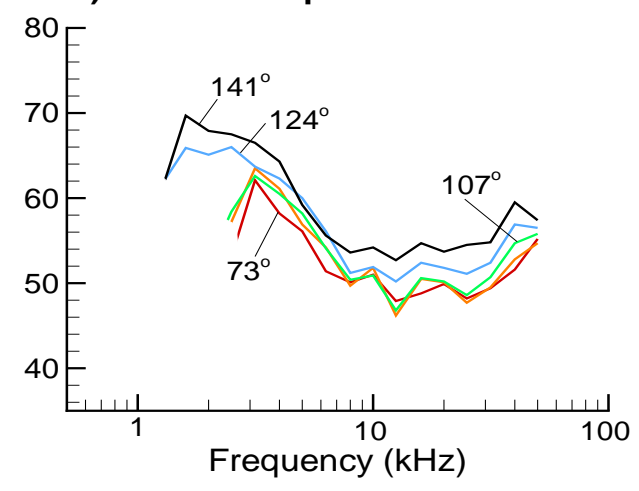

Figure 13. Cove-noise directivity one-third-octave band spectra for Mach 0.17.

\section{Conclusions}

An examination of the noise radiating from a CML flap is presented. Noise levels and source distributions are compared to that for a blunt flap. The DAMAS method was used to obtain noise spectra and spatial noise source distributions. The COP method was also used to identify noise source characteristics in the TE region of the CML. The results from the COP analysis identified significant contributions to the radiated field between 1 and $3.5 \mathrm{kHz}$. In that frequency range, the apparent noise source strength distributions obtained with the COP method indicate that the noise sources are distributed along the CML TE, and the locations of the strongest radiation are different for the pressure and suction sides.

DAMAS spectra revealed that the noise from the CML region is 5 to $17 \mathrm{~dB}$ lower (depending on flap deflection and Mach number) than the noise from the blunt flap side edge. The largest reduction is achieved for the $39^{\circ}$ flap configuration. The spectral analysis also revealed a peak in the noise measured from the cove region of the CML and blunt flap, in the higher frequency range. This peak corresponds to noise that is scattered at the TE of the main airfoil and channeled into the flap cove region. Application of serrated tape to the TE of the main element (in the region of the flap span) was relatively effective in reducing the cove noise spectral peak, but it strengthened noise sources at lower frequencies. These results establish the potential importance of main element TE geometry on the cove noise for flaps of longer span.

\section{Acknowledgements}

The authors wish to gratefully acknowledge Gerald E. Plassman and Larry L. Becker for data processing, software development, and analysis. The authors also gratefully acknowledge Daniel J. Stead, Dennis H. Kuchta, Jaye A. Moen and Ronald S. Geouge for invaluable support in all aspects of the experiment. 


\section{References}

1. Kendall, J. M.: Measurements of Noise Produced by Flow Past Lifting Surfaces, AIAA paper 78-238, 1978.

2. Block, P. J.: Assessment of Airframe Noise, Journal of Aircraft, Vol. 16, no. 12, pp. 834-841, Dec. 1979.

3. Athye, W. F., Miller, W. R. and Meecham, W. C.: Wing and Flap Noise Measured by Near and Far Field Cross Correlation Techniques, AIAA Paper 79-067, March 1979.

4. Fink, M. R. and Schlinker, R. H.: Airframe Noise Component Interaction Studies, Journal of Aircraft, Vol. 17, no. 2, pp. 99-105, Feb. 1980.

5. Kendall, J. M. and Athye, W. F.: Noise Generation by a Lifting Wing/Flap Combination at Reynolds Numbers to $2.8 \times 10^{6}$, AIAA Paper 80-0035, 1980.

6. Khorrami, M. R., Singer, B. A. and Takallu, M. A.: Analysis of Flap Side Edge Flow Field for Identification and Modeling of Possible Noise Sources, SAE Paper 971917, May 1997.

7. Meadows, K. R., Brooks, T. F., Humphreys, W. M., Hunter, W. W. and Gerhold, C. H.: Aeroacoustic Measurements of a Wing-Flap Configuration, AIAA Paper 97-1595, May 1997.

8. Radezrsky, R. H., Singer, B. A. and Khorrami, M. R.: Detailed Measurements of a Flap Side-Edge Flow Field, AIAA Paper 98-0700, 1998.

9. Khorrami, M. R., Singer, B. A. and Radezrsky, R. H.: Reynolds-Averaged Navier-Stokes Computations of a Flap Side-Edge Flow Field, AIAA Paper 98-0768, 1998.

10. Takallu, M. A. and Laflin, K. R.: Reynolds-Averaged Navier-Stokes of Two Partial-Span Flap Wing Experiments, AIAA Paper 98-0701, 1998.

11. Streett, C. L.: Numerical Simulation of Fluctuations Leading to Noise in a Flap-edge Flow field, AIAA Paper 98-0628, 1998.

12. Guo, Y. P.: Prediction of Flap Side Edge Noise, AIAA Paper 99-1804, 1999.

13. Brooks, T. F. and Humphreys, W. M: Flap Edge Aeroacoustic Measurements and Predictions, Journal of Sound and Vibration, Vol.261, pp.31-74, 2003.

14. Revell, J., Kuntz, H., Balena, F. Horne, C., Storms, B., and Dougherty, R.: Trailing Edge Flap Noise Reduction by Porous Acoustic Treatment, AIAA Paper 97-1646, 1997.

15. Storms, B., Ross, J., Horne, W., Hayes, J. Dougherty, R., Underbrink, J., Scharpf, D., and Moriarty, P.: An Aeroacoustic Study of an Unswept wing With a Three-Dimensional High-Lift System, NASA/TM-1998-112222, Feb. 1998.

16. Choudhari, M. and Khorrami, M.: Computational Study of Porous Treatment for Altering Flap Side-Edge Flow Field, AIAA Paper 2003-3113.

17. Slooff, J. W., de Wolf, W. B., van der Wal, H. M. and Maseland, J. E.: Aerodynamic and Aero-acoustic Effects of Flap Tip Fences, AIAA Paper 2002-0848.

18. Van der Wal, H. and Sijtsma, P.: Flap Noise Measurements in a Closed Wind Tunnel with a Phased Array, AIAA Paper 2001-2170.

19. Koop, L. and Dillmann, A.: Reduction of Flap Side Edge Noise Passive and Active Flow Control, AIAA 20042803.

20. Koop, L., Ehrenfried, K. and Dillmann, A.: Reduction of Flap Side Edge Noise by Active Flow Control, AIAA 2002-2469.

21. Hutcheson, F. and Stead, D.: PIV Measurements on a Blowing Flap, AIAA Paper 2005-0212.

22. Streett, C, Casper, J., Lockard, D. Khorrami, M., Stoker, R., Elkoby, R., Wenneman, W. and Underbrink, J.: Aerodynamic Noise Reduction for High-Lift Devices on a Swept Wing Model, AIAA paper 2006-212.

23. Storms, B., Hayes, J., Jaeger, S. and Soderman, P.: Aeroacoustic Study of Flap-Tip Noise Reduction Using Continuous Moldline Technology, AIAA Paper 2000-1976.

24. Liebeck, R., Page, M. and Rawdon, B.: Blended-Wing-Body Subsonic Commercial Transport, AIAA paper 980438.

25. Brooks, T. and Humphreys, W.: A Deconvolution approach for the mapping of Acoustic Sources (DAMAS) Determined from Phased Microphone Arrays, Journal of Sound and Vibration, Vol. 294, pp. 856-879, 2006.

26. Humphreys, W. and Brooks, T.: Noise Spectra and Directivity For a Scale-Model Landing Gear, AIAA paper 2007-3458.

27. Bendat, J. and Piersol, A.: Engineering Applications of Cross-correlation and Spectral Analysis, $2^{\text {nd }}$ Edition, 1993, Wiley, New York, 1980.

28. Humphreys, W. and Brooks, T., Hunter, W. and Meadows, K.: Design and Use of Microphone Directional Arrays for Aeroacoustics Measurements, AIAA paper 98-0471. 
29. Brooks, T. and Hodgson, T.: Trailing Edge Noise Prediction from Measured Surface Pressures, Journal of Sound and Vibration, Vol. 78 (1), pp.69-117, 1981.

30. Brooks, F. and Humphreys, W.: Extension of DAMAS Phased Array Processing for Spatial Coherence Determination (DAMAS-C), AIAA-2006-2654.

31. Mendoza, J., Brooks, T. and Humphreys, W.: Aeroacoustic Measurements of a Wing/Slat Model, AIAA paper 2002-2604.

32. Hutcheson, F. and Brooks, T.: Effects of Angle of Attack and Velocity on Trailing Edge Noise, AIAA paper 2004-1031. 